\title{
Why brain banking should be regarded as a special type of biobanking: ethical, practical, and data-management challenges
}

This article was published in the following Dove Press journal:

Journal of Biorepository Science for Applied Medicine

12 May 2015

Number of times this article has been viewed

\author{
Sara Y Nussbeck $k^{1,2, *}$ \\ Wiebke MWemheuer $3,4, *$ \\ Katharina Beier ${ }^{5}$ \\ 'Department of Medical Informatics, \\ University Medical Center Göttingen, \\ Göttingen, Lower Saxony, Germany; \\ 2UMG Biobank, University Medical \\ Center Göttingen, Göttingen, Lower \\ Saxony, Germany; ${ }^{3}$ Department of \\ Neuropathology, University Medical \\ Center Göttingen, Göttingen, Lower \\ Saxony, Germany; ${ }^{4}$ Experimental \\ Neurobiology Group, Luxembourg \\ Centre for Systems Biomedicine, \\ University of Luxembourg, \\ Luxembourg; ${ }^{5}$ Department of Medical \\ Ethics and History of Medicine, \\ University Medical Center Göttingen, \\ Göttingen, Lower Saxony, Germany \\ *These authors contributed equally to \\ this work
}

Correspondence: Katharina Beier Department of Medical Ethics and History of Medicine, University Medical Center Göttingen, Humboldtallee 36, 37073 Göttingen, Lower Saxony, Germany $\mathrm{Tel}+49551394184$

Fax +49 55I 399554 Email katharina.beier@medizin.unigoettingen.de
Abstract: Biobanking of the brain and other central nervous system materials, ie, brain banking (BB), provides an important research tool for understanding the causes of neurodegenerative and psychiatric diseases. Particularly with aging societies, there is an increasing need for molecular neuropathological research in this field. While there is an extensive debate on biobanking in general, the specific challenges that the procurement, processing, and storage of postmortem human brain tissue, and especially whole brains, raise are hardly ever considered systematically. This paper analyzes the peculiarities that make BB a distinct type of biobanking by combining the perspectives of neuropathology, medical informatics, and medical ethics. While ethical, practical, and data-management issues are often dealt with separately and the focus of such research is on only specific aspects of BB, this paper aims at an integrated analysis of the whole process. Six crucial steps in the BB workflow are analyzed: a) donor recruitment, b) follow-up during the donor's lifetime, c) postmortem brain donation, d) neuropathological diagnosis, e) research with brain tissue, and f) the provision of brain material to third parties. A comprehensive understanding of the challenges that $\mathrm{BB}$ raises is vital for making this practice more effective but also to counteract the current decline in brain-donation rates.

Keywords: brain autopsy, brain donation, neuropathology, neuroscience research, ethical, legal, and social issues, IT infrastructure

\section{Introduction}

"Biobanking" describes all of the professionalized processes involved in the organized storage and provision of biomaterials that are linked to donors' phenotypic and clinical information. ${ }^{1,2}$ Biomaterial itself can be classified into liquids, solids, and cells. Ethical, ${ }^{3,4}$ practical, ${ }^{5}$ and information technology (IT) issues ${ }^{6-8}$ arise throughout biobanking. While these debates usually focus on biobanking in general, this paper focuses on the practice of brain banking (BB) as a distinct type of biobanking.

$\mathrm{BB}$ is the biobanking of central nervous system (CNS) tissue (eg, whole brains, brain biopsies, and spinal cord samples) and associated liquids, eg, cerebrospinal fluid (CSF) and blood. While CSF and small CNS tissue samples can be obtained from a living patient the procurement of whole brains from diseased and healthy people requires their death. ${ }^{9,10}$ Postmortem brain donation is indispensable for neuropathological research, but it also raises certain questions that distinguish the procurement, processing, and storage of human brain tissues from the biobanking of other tissue specimens. Whole brains provide the basis for research that aims to explain complex brain disorders like neurodegenerative diseases. Animal models that resemble these kinds of diseases are useful, but they cannot replace human brain material derived from 
patients who have suffered from brain diseases. As the BB of CSF and blood does not differ greatly from the biobanking of other liquids, our analysis will focus on the banking of solid tissues and, particularly, of whole brains.

With the global trend of aging societies, health care costs will rise at an increasing rate in the coming decades. ${ }^{11}$ A study by the European Brain Council estimated that 127 million Europeans were affected by brain disorders in 2004 which summed up to a cost of approximately 400 billion Euros for the health systems. ${ }^{12}$ In an aging society, neurodegenerative diseases in particular will occur more frequently. Research on brain material is necessary if we are to understand better, treat, and prevent brain disorders in the future. Most present brain collections are disease-specific focusing on, eg, Parkinson's disease, HIV, or a family of conditions like psychiatric diseases. ${ }^{13-15}$ But there are also brain banks which collect brains of various disease entities as well as healthy brains, though many fewer. Some brain banks are very large, for example, the Netherland's Brain Bank has collected more than 3,700 brains since 1985, ${ }^{16}$ and the New York Brain Bank holds the brains of more than 5,000 donors. ${ }^{17}$

Due to the scarcity of brain donors, the trend is towards networking, eg in 2001, the European Commission launched BrainNet Europe, ${ }^{18,19}$ an international consortium of 19 brain banks throughout Europe. Efficient networking requires the standardization of workflows, diagnostic procedures, and data acquisition so that samples and data are comparable. The distinctiveness of BB in the context of research biobanking is occasionally stressed by pointing out the differences between the donation of brains and other tissues/organs. ${ }^{20,21}$ Although several recommendations for the operating of research brain banks have been issued, ${ }^{18,19,21-23}$ no globally accepted standard protocol or regulations for banking brain tissues are in place. ${ }^{22,24}$ With regard to BB's ethical issues BrainNet Europe has published a Code of Conduct to the partners of the consortium. ${ }^{25,26}$

While there is an extensive debate on biobanking in general, the specific challenges that the procurement, processing, and storage of postmortem human brain tissue, and especially whole brains, raise are hardly ever considered systematically.
This paper analyzes the peculiarities that make $\mathrm{BB}$ a distinct type of biobanking by combining the perspectives of medical ethics, neuropathology, and medical informatics. While ethical, practical, and data-management issues are often dealt with separately ${ }^{27}$ and the focus of such research is on only specific aspects of BB, this paper aims at an integrated analysis covering the whole process. Six essential steps in the BB workflow are analyzed: a) donor recruitment, b) follow-up during the donor's lifetime, c) postmortem brain donation, d) neuropathological diagnosis, e) research with brain tissue, and $f$ ) the provision of brain material to third parties (Figure 1). An integrated understanding of the challenges $\mathrm{BB}$ raises is vital not only for making the practice more effective but also to counteract the current decline in brain donation rates.

\section{Challenges}

\section{The recruitment of donors}

Today the recruitment of brain donors is increasingly organized via antemortem donation programs (DPs) in which donors (usually patients diagnosed with neurodegenerative or psychiatric diseases but also healthy volunteers) are enrolled with a brain bank before their death. From an ethical perspective, the advantage of antemortem DPs lies in the early interaction they bring about between brain banks (ie, a brain bank's staff, especially neuropathologists but also researchers) and the public, ${ }^{28}$ ie, patients, healthy donors, and patient organizations. From the perspective of research, DPs are useful because they allow for the continuous standardized collection of medical data and samples (eg, CSF, blood, and biopsied tissue) during a donor's lifetime. Furthermore, they facilitate quick autopsies after which any data obtained during the course of a disease can be compared with postmortem neuropathological results.

When enrolling a donor, it is essential to document their preliminary diagnoses, particularly when contagious diseases are suspected. Similarly, the potential donor's physician should communicate tentative diagnoses to attending specialists and the relevant surveillance units. For example, a physician's failure to communicate a tentative diagnosis of Creutzfeldt-Jakob disease (CJD) may lead to instruments

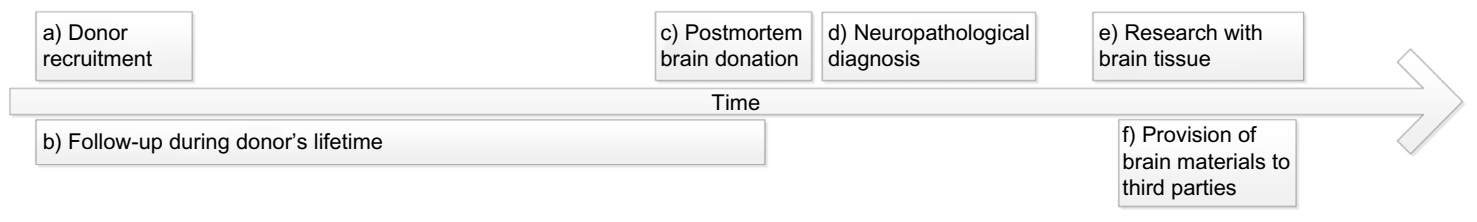

Figure I The six steps in brain banking. 
becoming contaminated with prions when surgery or the autopsy is performed which, in turn, endangers staff and other patients because the standard decontamination procedure does not always inactivate prions. Thus, especially thermolabile instruments, like endoscopes, which are frequently used for percutaneous endoscopic gastrostomy in demented, bed-ridden patients, may need special reprocessing. ${ }^{29}$

Recruitment also poses IT challenges. A DP must document donors' medical data, contact information (and that of their next of kin), and the options listed on the consent form that donors have chosen, for example, to allow genetic testing or be informed about incidental findings. And because brain donation can occur a long time after recruitment, a DP must keep this information up to date. A study-participant management system is an IT tool for managing contact and informed consent information. ${ }^{30}$ It can keep track of donors' contact history, current contact details and those of their next of kin, and their informed consents and withdrawals of consent.

Brain material from healthy donors is indispensable as controls for understanding the causes of neurodegenerative and psychiatric disorders. Few people realize how urgently brains from healthy donors (which serve as controls) are needed. Therefore, brain banks often reach out to the spouses or relatives of patients, ${ }^{20,27}$ who are more willing to donate their brains for research than those in the average population. ${ }^{31}$

Another challenge for $\mathrm{BB}$ is the decline in autopsy rates. ${ }^{20}$ Rates are declining because current legal regulations pose obstacles to brain autopsies and physicians today are hesitant to make the next of kin uncomfortable by asking them for permission to perform an autopsy. ${ }^{32}$ One might think that this discomfort results, at least partly, from the brain's special significance (ie, our view of it influences our understanding of ourselves and others). ${ }^{33}$ Therefore, brain donation raises ethical questions that do not arise for other sorts of donation. ${ }^{34}$ Although this "brain exceptionalism" is challenged by studies that show that many people do not fundamentally distinguish the donation of a brain from that of other organs, ${ }^{33,35}$ other studies reveal sensitivities toward the former. For example, one study showed that the majority of respondents are less troubled by the obtainment of brain tissues than by the extraction of the whole brain. ${ }^{36}$ Moreover, since the brain sits in the head, brain donation raises fears of disfigurement. ${ }^{37}$ Finally, in the case of psychiatric disorders that are particularly stigmatizing, potential donors may be uncomfortable with the prospect of becoming connected with a brain bank specializing in such diseases, even if they do not suffer from any of them. Consequently, brain samples are more difficult to acquire than other tissue specimens. ${ }^{38}$
$\mathrm{BB}$ also raises its own challenges for obtaining informed consent. In 2003, the Isaac's Report revealed that several thousand brains had been collected in the UK without familial consent. ${ }^{39}$ This resulted in a revision of the Human Tissue Act (HTA) requiring the next of kin's consent for autopsies and the removal of organs and tissues for research. ${ }^{19,40} \mathrm{~A}$ second challenge concerns the very possibility of consent for a person whose understanding is seriously impaired by psychiatric or neurodegenerative disease (eg, schizophrenia and Alzheimer's disease).$^{40}$ While research ethics recognizes special protections for persons incapable of giving consent, their participation is permitted if the research in question is likely to benefit future patients with the same disease. Consent can then be given by the patient's legal representative. ${ }^{41,42}$ However, for brain donation with proper planning the legal necessity of getting the representative's consent can often be avoided. Since in many neurodegenerative diseases cognitive and emotional capacities diminish over time, such patients may be recruited while they are still competent. Moreover, psychiatric patients may be capable of informed consent in the absence of acute depression or psychosis. ${ }^{20,43}$ Thus, in asking for consent, timing is important, as it is for the patients themselves who must be given sufficient time, given their conditions, to decide about brain donation.

For biobanking in general, consent is required to be maximally specific, though in practice this is often unachievable. For this reason, if an envisioned research project is not within the scope of the prior consent and attaining new consent is not possible with reasonable efforts, evaluation by an ethical review board is the default. ${ }^{44}$ In the biobanking of other tissues, a renewal of the donor's consent remains at least theoretically possible, but postmortem BB excludes this option by its very nature. For this reason, and also because new requests may be stressful for the next of kin, it is particularly important in BB to ensure that the donor's and his next of kin's initial consents are sufficiently comprehensive and cover the most recent analytical methods, eg, whole genome sequencing and other high throughput measures in order to avoid any later lack of permission for research. Most importantly, the framework of even broad consent requires that an independent ethics committee reviews each research project. $^{45}$

\section{Following up on donors during their lifetimes}

DPs usually approach patients with the onset of clinical symptoms. Thus, the time from recruitment to donation can be from weeks to months on average for a patient with CJD and up to 
several decades for patients suffering from multiple sclerosis (MS) and healthy donors. Such long time spans create several challenges, especially for IT. For one example, follow-up data on donors who move or change their names must be correctly recorded. Therefore, IT-management systems need robust de-coding algorithms that allow for error correction. ${ }^{46}$ For instance, two patients whose surnames are recorded as Schmitt and Schmidt and who have identical first names and dates of birth listed are probably the same person, and an adequate algorithm is able to detect this and alert the person in charge of data entry. ${ }^{6,7}$ For another example, different clinicians, eg, neurologists and psychiatrists from private practices and specialized clinics, may be involved in treatment over several years, and comprehensive documentation of clinical data might therefore be difficult to obtain and the anamneses very heterogeneous. One solution for overcoming heteroanamnesis and ensuring high-quality data collection over a long period of time is to enroll the patient into a DP as early as possible. Finally, for BB to be efficient, data from medical reports of diagnoses and disease progression must be entered into a central database, but even if the data are available, these reports are not usually standardized. Ideally, clinical studies on CNS diseases are linked to DPs and regularly collected medical data are directly transferred to the data base of the DP.

There are five IT issues about long-time archiving. Firstly, standards for diagnostic procedures and criteria within research areas can change over time. The diagnostic criteria for MS, for example, changed twice within the last decade. ${ }^{47}$ Thus, a preliminary decision on any metadata standards for describing clinical and imaging data must be made. Secondly, given the long time spans, steps must be taken to ensure that data remain available beyond the lifespan of the present IT system. The third issue involves data formats. If reading or visualizing any data requires a certain software application, their formats are probably proprietary (especially if they are image data), and this could mean that the same version of the software will be needed, decades later, to read the data. For this reason, software needs to be archived. Fourthly, in addition to the technical issues with data availability and accessibility, a model of incentives for physicians to submit their patient data to a central database should be developed. An alternative method is patient-reported outcomes in which patients record the progression of their own diseases. ${ }^{48}$ Although these data might not be reliable ${ }^{49}$ after a certain stage in a disease's progression, they can still be valuable additions to physicians' medical reports. Lastly, IT must deal with changes in dataprotection requirements over time. This means that in addition to continuous IT support, new investments in software and hardware about every 5 years are necessary for sufficient data management throughout the follow-up time.

The need for well-characterized materials that are linked to a patient's medical records puts several strains on patients and their families. For example, due to follow-up examinations, patients, and also healthy donors, become study subjects during their lifetimes. Moreover, the meticulous documentation of each step of deterioration will constantly remind patients and their families of their disease's irrevocability. Next of kin may also feel distress in the case of heritable diseases. Life-long supervision by a brain DP also raises issues of privacy and confidentiality. ${ }^{43}$ The continuous flow of data from the patient's attending doctor to the surveillance unit or brain bank can be a delicate matter in the case of stigmatizing diseases.

\section{Postmortem brain donation}

From the research point of view, the postmortem time (PMT) should be as short as possible (ideally less than 24 hours) as this ensures tissue of high quality. In any case, PMT should be documented for the sake of sample comparability. The required short time interval from death to autopsy brings ethical and, occasionally, organizational challenges (if the corpse has to be transported over a long distance to the neuropathology unit). Particularly in the case of unexpected death, BB depends on the next of kin's readiness to contact the brain bank immediately and provide the necessary documents. As this must be done at a time of intense emotional stress, several brain banks provide detailed instructions and give potential donors brain-bank donor cards. ${ }^{50}$

The bereaved may also experience stress when the deceased was not enrolled in a DP and did not articulate his/her wishes. As autopsies for research purposes require relatives' consent in most countries, prior contact with them might be useful. Some brain banks contact the next of kin after death via telephone, ${ }^{19}$ which can be too much of a burden. There is a trade-off in timing between sensitivity and research requirements: the longer after death the request for brain donation comes, the higher the likelihood of donation ${ }^{35}$ but the worse the quality of the tissue. It is important to explain to the next of kin that the autopsy will not enhance the changes taking place after death in the body of the deceased. It is due to those biochemical processes that the corpse of someone once dear is perceived as increasingly foreign as time passes whether or not an autopsy has been performed. When professionals take the family's concerns seriously and provide adequate counseling (ideally involving both clinical and research teams) the 
next of kin's support for donation can be obtained in the majority of cases. ${ }^{35,36,51}$

From an ethical perspective, the donor's next of kin plays a similar role in the context of brain donation for research as in organ donation for transplantation. While the next of kin's permission is not legally required when the deceased had already agreed to the donation, in practice, family members are given a right of veto (Table 1). ${ }^{52}$ Thus, as BrainNet Europe's Model Information Leaflet states, "Please note that regardless of the donor's wishes, the autopsy will not be carried out if the family resolutely refuses it". ${ }^{53}$

In fact, the next of kin's position after the donor's death is especially strong in the context of brain donation. ${ }^{10}$ Although in Germany, for example, the next of kin, as the patient's legal representative, is expected to decide about tissue/organ donation on the basis of the patient's presumed will, ${ }^{54}$ no brain donation will occur if the family resolutely refuses. In the UK, the HTA Code of Practice on Consent does not mention any criteria for the representatives' choices ${ }^{10}$ but allows (in contrast to the general provisions of the HTA) that in order to avoid traumatizing family members ${ }^{55}$ the next of kin's own wishes may overrule the deceased's. The proper balance between the donor's decision and its impact on his/her next of kin is currently a subject of discussion..$^{40}$ For example, some question whether the family's wishes should be allowed to overrule the deceased's given that the donor has the right that his/her wishes be respected ${ }^{18}$ and that brain research contributes to the common good of health. ${ }^{10}$

Once the family has agreed, a neuropathology team removes and processes the brain tissue. The assortment, fixation, and storage of the samples depend on the tentative diagnosis and the research aim. The tentative diagnosis also determines whether additional tissue, like the spinal cord (whose removal is more complicated than the brain's), is taken. Ideally, additional extra-cerebral tissues (from the eyes, plexus and ganglia, and parts of bodily organs) are obtained during autopsy. This, however, might exceed the calculated work time and storage space. Therefore, standards for particular diseases and projects are best defined in advance.

Biomaterial annotation data, which describe all of the steps - excision, processing, and storage - performed on

Table I Brain donation in relation to donor's and family's desires

\begin{tabular}{lll}
\hline Donor's desire & Next of kin's desire & Consequence \\
\hline No donation & No donation & No donation \\
No donation & Donation & No donation \\
Donation & No donation & No donation \\
Donation & Donation & Donation \\
\hline
\end{tabular}

the brain material and include the unique coding of every sample, its exact storage location, and any information on its quality that is available, are then collected. This creates special problems as the coding of samples depends on the way a brain is processed, which may differ from disease to disease.

In this context, especially the misuse of anatomical terms, hinders efficient sample distribution upon request. ${ }^{56}$ Another annotation problem is the mapping of small pieces of tissue removed from single brain slices to their locations in those slices and the subsequent comprehensive coding. ${ }^{57}$ Thus, BrainNet Europe has standardized the samples to be taken postmortem ${ }^{58}$ and their coding for psychiatric diseases. Unfortunately, such standards/standard operating procedures do not exist for all diseases and are urgently needed. ${ }^{21,27,56}$ Vonsattel et al, for example, mention nine different protocols for the processing of fresh brain tissue. ${ }^{56}$ Moreover, accompanying data sets and defined minimal data sets for specific diseases collected by brain banks are extremely difficult to identify in the literature. For sample and data exchange, however, the former are of the uttermost importance, and scientists should be encouraged to publish their data sets.

\section{The neuropathological diagnosis and its consequences}

The recommended first step in diagnosing a brain biopsy from a patient with dementia is to exclude prion diseases (the most common of which is sporadic CJD). ${ }^{59}$ The same should apply to autopsy-derived whole brains. During the dissection of a brain, the neuropathological diagnosis has not been made yet, so the neuropathologist must assume maximal infectiveness of brain tissue, CSF, and blood. This also applies to other contagious diseases, eg, tuberculosis, hepatitis, and $\mathrm{HIV}$, in addition to potential prion infection. ${ }^{60}$ In contrast to most viral and bacterial contaminants, however, alcohol and formalin do not inactivate prions. Consequently, even paraffin-embedded tissue blocks from a patient with a prion disease can still be infectious. In such a case, tissues need to be decontaminated with formic acid before the embedding process. ${ }^{61}$ Whether decontamination is necessary can be determined by an established biochemical workflow, which detects pathological prion protein in native samples with high sensitivity and specificity, while the rest of the brain or biopsy is fixed in formalin. ${ }^{62}$ The routine use of formic acid is not advisable as it destroys many epitopes that are important for further diagnostic inquiries. So, to prevent contamination by infectious agents, fresh brain sections about to be frozen should be sealed in plastic bags whose outsides have not come 
into contact with any brain tissue. Freezing brain slices or sections between metal plates ${ }^{63}$ is an elegant way to produce flat, frozen slices of good quality, but the tissue must be placed in plastic bags first. Afterward, tissues infected with prions require either decontamination, as described above, ${ }^{61}$ or exclusive handling in $\mathrm{S} 3 *$ units.

Neuropathological diagnosis uses histopathology and immunohistochemical staining techniques. If required, electron microscopy or double fluorescence staining techniques can also be used. If infectious agents, like viruses or bacteria, are suspected to have caused neuroinflammation, confirming evidence can be obtained on the genomic level by polymerase chain reaction or in situ hybridization. As a general trend, the diagnosis of brain diseases continually becomes more complex as new methods are developed and conventional ones refined. In order to make BB across national borders within Europe and in worldwide networks, possible/effective, similar or at least comparable standards should govern diagnostic methods. Proficiency testing will benefit the progress and availability of diagnostic methods. The increased work force and use of precious materials has to be balanced against improvements in the diagnostic procedure. ${ }^{64}$ The same applies to the question whether diagnostic standards for each case should exceed the actual diagnosis, eg, by quantifying protein aggregates, such as $\alpha$-synuclein, for specific neuroanatomic areas in cases of dementia with Lewy bodies. Such information, in addition to diagnoses, could improve the selection of patient tissue for future research projects and, thus, prevent the waste of valuable material.

In neurodegenerative diseases, an astonishingly large proportion $(15 \%-35 \%)$ of antemortem diagnoses will prove wrong or incomplete upon postmortem brain-tissue analysis. ${ }^{65}$ In cases where clinical and neuropathological diagnoses differ, both must be documented. It would be interesting to analyze these cases in order to find out why different diagnoses were initially considered. Regardless of the reasons, however, this fact implies that clinical studies include patients who should not have participated. ${ }^{66,67}$ Brain autopsies would allow researchers to exclude such patients from the evaluation of clinical studies with the benefit of hindsight and therefore make studies more precise in smaller case numbers. This is an argument for enrolling patients with neurodegenerative or psychiatric diseases in DPs via surveillance programs or clinical trials.

Providing relevant information to families is a particularly sensitive ethical issue, as several neurodegenerative and psychiatric diseases have a genetic basis and, thus, may affect family members. In general, the disclosure of information about the deceased should be decided case by case. ${ }^{55}$ In line with this, brain banks in BrainNet Europe disclose information only on the family's request. Moreover, the communication of research results is restricted to "very exceptional cases, when information that emerged in the course of research is of obvious clinical importance to surviving relatives". In such cases, "the acquired confidence with the donor may become irrelevant and the possibility of giving information about new findings should be taken into account". ${ }^{53}$ The early involvement of family members in the decision about brain donation can help prevent cases where a donor does not allow disclosure but his next of kin desires it. Ethical counseling before and after any release of information, particularly genetic information, is indispensable. The data-management system must ensure that relatives have the option to know or not to know about a clinically relevant condition, and it must also be able to administer changes of attitude on this matter over time. This will be particularly important as new associations between genes and diseases are established in the future. ${ }^{39}$

\section{Requirements for research with brain tissue}

Proper specimen storage is indispensable if donated brain tissue is to be available for years or decades. This requires that, as with all other biobanked material, tissues be preserved in ways that make them suitable for high-quality biochemical analyses and histopathology. Therefore, certain parts of the brain need to be stored at $-80^{\circ} \mathrm{C}$ while others must be fixed (usually in 10\% formalin, which equals $4 \%$ formaldehyde) for histology techniques. A sensible method is first to freeze the securely bagged sliced brain sections between cooled metal plates and then store them in labeled, stackable packaging. Already at this point the protocols of different brain banks reveal a broad range of variation: while the protocol of the National NeuroAids Tissue Consortium requires the freezing of infectious brains in coronal slices, ${ }^{68}$ the Edinburgh Brain Bank freezes a selection of tissue fragments for HIV-infected cases but prefers to freeze larger tissue blocks or cerebral and cerebellar hemispheres for CJD cases. Naturally, large tissue blocks require extensive post-processing. However, Columbia University has developed an elaborate dissection protocol for fresh brain hemispheres that results in up to 150 samples, as either tissue blocks or pulverized aliquots, that can easily be provided upon request without further post-processing. ${ }^{63}$

The parameter that determines the quality of frozen tissue samples is the $\mathrm{pH}$ value; it directly correlates with RNA quality, which can be assessed by determining the total RNA 
yield per unit and the extent of degradation of ribosomal RNA. ${ }^{22,63}$ RNA quality and $\mathrm{pH}$ depend not so much on the PMT as on the agonal state of the patient when he died; this has to be kept in mind when matching control cases for molecular research.

Fixed tissues can be stored in three ways: in containers of fixative, as paraffin-embedded tissue blocks, and as cut tissue sections on glass slides or membranes. The steps in preserving CNS tissue by fixation partially overlap with the processing of the brain and depend on a reliable diagnosis. The outsides of formalin containers must not be contaminated, so brain hemispheres or slices should be put into the containers with great care. An advantage to keeping large portions of a brain in formalin is that specific neuroanatomical areas can be dissected according to the wishes of the requesting researcher. A clear disadvantage of the fixation method is that certain epitopes for immunohistochemical detection are lost (some as early as 20 days after fixation has begun). If formalin-preserved tissue is post-processed for research purposes, it is advisable to verify its present quality with established immunohistochemistry protocols and controls that provide information about the degradation of specific epitopes.

Sufficient space must be available for the long-term storage of specimens, ie, enough for formalin-fixed brains, paraffin blocks, and frozen material. Freezers and liquid nitrogen tanks must be adequately secured against power failure and come with an alarm system and an emergency plan in case of break down. This is a crucial, if obvious, point because accidental freezer thaws can set brain research back many years, as happened with Harvard's McLean Brain Bank for autism. ${ }^{69,70}$

The identification of suitable brain tissues for a given line of research is essential for researchers. Besides clinical parameters, researchers should ensure that the envisioned research is covered by the donor's and the next of kin's consent, for returning to a family for renewed consent is ethically problematic. ${ }^{18}$ To enable searching for suitable samples in any disease area, BrainNet Europe published a standard ${ }^{18}$ that includes research consent status, age, sex, cause of death, salient clinical and drug history, agonal state, postmortem interval, neuropathological diagnosis, and a list of the tissue samples available. However, there are two problems with the standard. First, it is unclear whether this minimal dataset is available for every brain at every brain bank in Europe. The second problem is the lack of a standardized nomenclature allowing for effective searches in a common database. With, for example, the cause of death, the use even of medical synonyms should be avoided. If there is no underlying mapping of terms, the database cannot classify heart attack and cardiac infarction as the same cause. Although this problem is not unique to $\mathrm{BB}$, it can cause special problems here because the donors of whole brains, who could have answered questions regarding medication history or disease progression, are no longer available.

\section{Providing brain material to third parties and financial sustainability}

Ethically, it is crucial to get the maximum research benefit from stored brain tissue. ${ }^{28}$ Beyond the general scarcity of brains, some valuable brain parts are in especially short supply, which raises the challenge of a just allocation among researchers. Intelligent processing of brain material may (depending on the size of the relevant brain regions) solve part, but only part, of the problem. Thus, it is important to develop a policy of equitable tissue distribution in BB based on safety, ethical principles, and scientific excellence. ${ }^{18}$ A standardized procedure in which the board of a brain bank, supported, if necessary, by an ethical review board, reviews any request for the release of brain material could strengthen public confidence in $\mathrm{BB}$.

The prioritizing of samples lies within the expertise of neuropathologists as do the extensive fresh dissecting before storage and the post-storage processing of neuroanatomical sites that must be dissected from larger tissue sections. Mounting paraffin-embedded tissue sections onto slides or membranes requires additional qualified workers (usually technicians) which raises the cost of BB. Additional costs in $\mathrm{BB}$ accrue due to prolonged follow-up periods in some diseases. ${ }^{71}$ In general, cost is a sensitive issue in this field. Given the wide agreement that, in order to honor donors' altruism and ensure that the use of brain tissue is restricted to appropriate research, brain banks should not be commercial, ${ }^{20,21,28}$ they must be sustainably financed. However, as public funding of some networks (eg, BrainNet Europe) has expired, ${ }^{18}$ it is feared that financial, rather than scientific, interests will increasingly govern access to brain material. At the same time, though, the involvement of private stakeholders may be indispensable to covering the high cost of biobanks and brain banks in particular. Due to the labor-intensive pre-storage processing of brain material, the cost per brain for aquisition, processing, and storage runs between $€ 10,000$ in Europe and \$15,000 in the US and Asia. ${ }^{22}$ Although the principle of the non-commercialization of human body parts is widely advocated, adherence to the principle in practice seems to be less strong. ${ }^{72}$ Rather than immediately banning 


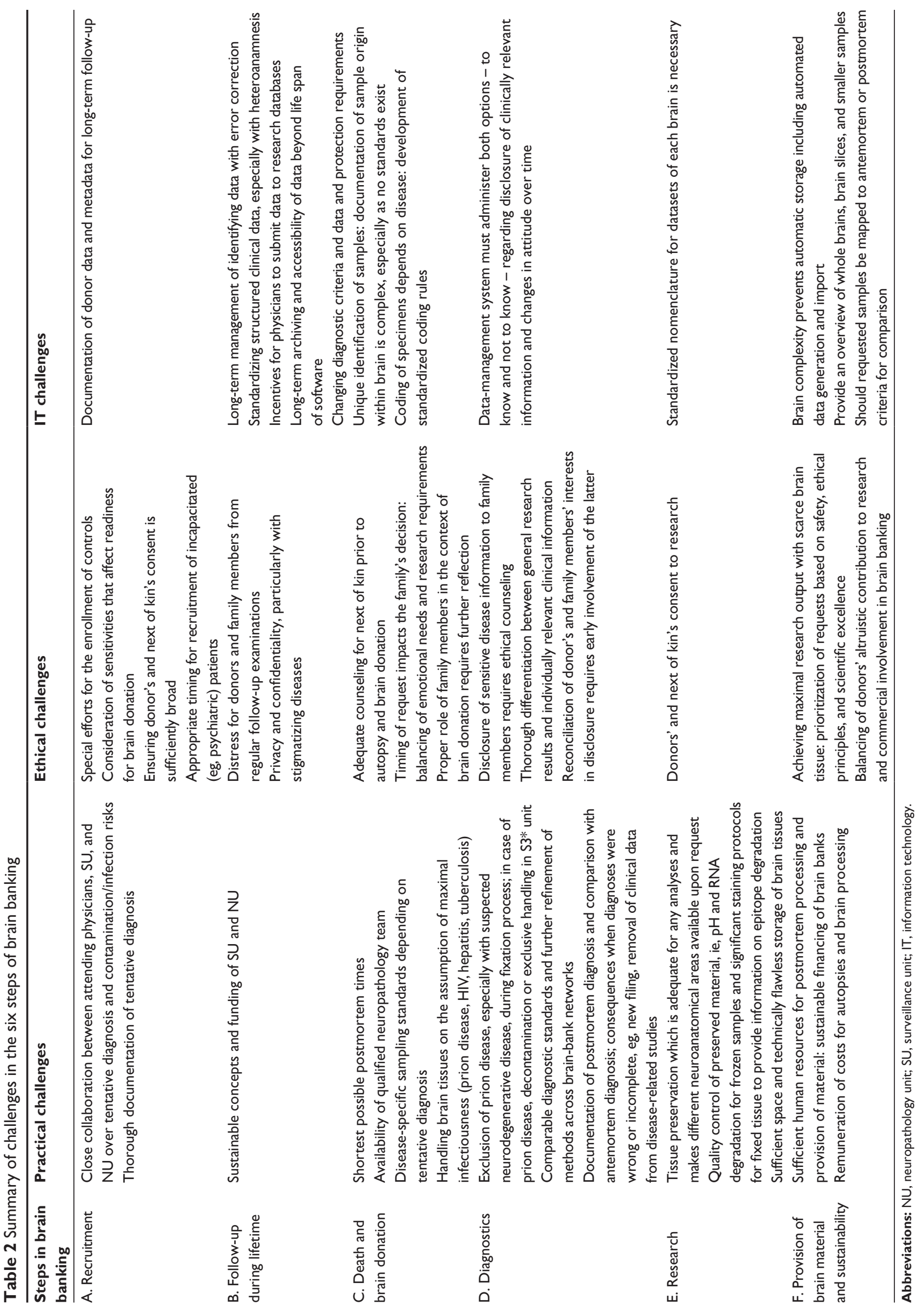


commercial interests, what is needed is a critical discussion over whether the involvement of private stakeholders will lead to financial dependencies that could hamper research in the public interest. ${ }^{28}$ Charitable trusts are currently seen as a promising way to avoid this. ${ }^{21}$

The financial sustainability of $\mathrm{BB}$ includes adequate remuneration of the costs involved in obtaining, processing, and storing tissues properly. ${ }^{21}$ These costs are often not taken into account in calculating user fees. An appropriate reimbursement scheme might either directly finance a neuropathologist at a brain bank or reimburse the neuropathology department in charge of sample processing. The latter would require appropriate budgeting when applying for research grants for projects involving CNS samples. The calculation of costs may need to take access, processing, and administration fees into account. Particularly when fees are charged for the processing of brain tissues, a detailed, digital documentation of the work to be conducted will facilitate cost calculations. The high cost of $\mathrm{BB}$ again highlights the importance of brain-bank networks that serve a broader research community.

BB differs from most other kinds of biobanking because of the necessary post-storage processing of brain tissue. The alternative of extensive pre-storage processing is similarly elaborate. ${ }^{63}$ Because the complexity and size of the brain makes it currently impossible to store samples in storage robots or process them automatically, post-storage processing also involves several challenges for IT. Though one can easily query data from single liquid and small tissue samples in any database, searching for brain samples is more difficult as a treasured neuroanatomical structure or pathologically altered area (eg, an MS lesion) can stretch across more than one brain slice. Moreover, there is a trade-off for pathologically altered brain material, between the need for specific data on the material and its further availability for research. Information on whether a whole brain or brain slices contain the desired area will be in a database only if the brain section containing the area has already been processed. However, if a brain section has been processed, then the desired neuroanatomical or pathologically changed area may no longer be available, or at least not completely, to other researchers. Therefore, current best practice is to provide an overview of the samples and types of preservation, eg, left hemisphere in formalin, right hemisphere cryo-preserved. For neuroanatomical structures, it might be an option to indicate whether they are still intact or have already been processed. In the latter case, a neuropathologist would have to determine whether a request could be fulfilled or not. Further available clinical and neuropathological data should also be included in the database.
Another important issue in BB is whether requested specimens should be mapped to antemortem or postmortem criteria for comparison between cases. ${ }^{22}$ The challenge for the IT system is to filter for both approaches in searching for suitable samples in a brain bank.

Imaging is one solution to the limited availability of brain tissue, and it has already been established in the clinical setting at some institutions. The two imaging options are virtual microscopy/digital pathology and brain atlases. The former involves scanning slides of the available brain regions with high-resolution microscopes. ${ }^{73}$ The images can then be provided upon request. The procedure greatly increases the work of each autopsy, but it can help neuropathologists make their requests for tissues appropriately specific, and it allows a direct comparison of immunohistochemical stainings from the providing and the receiving facilities. However, it cannot satisfy researchers' demands for special tissues, especially not for biochemical analyses. As for brain atlases, different ones, with different methods ${ }^{74,75}$ and disease foci, exist in digital formats, ${ }^{76}$ but their use in new research is limited.

As regards data protection and rules for clinical data access, there is no fundamental difference between BB and biobanking in general. However, given that the former can involve stigmatizing diseases and that data-protection laws are often limited to living persons, postmortem BB might require additional safeguards. For example, it is important to keep identifying patient data confidential even after a person's death. In addition, in accord with the principle of data minimization, researchers, including third parties, ought to receive only de-identified data when they are indispensable for research. Material (and data) transfer agreements usually set out detailed provisions for the release of samples and data to third parties that cover, eg, intellectual property rights, the scope of informed consent, and the return of data and materials.

\section{Summary}

In summation, BB differs from biobanking of other tissues in several ways. The ethical, practical, and IT challenges for the six steps of BB are summarized in Table 2.

\section{Conclusion}

Based on this paper's analysis of the distinctive features of $\mathrm{BB}$ in contrast to biobanking in general, there are at least four issues that require further discussion. Firstly, BB is a long-term practice. Depending on the illness, brain donation can take decades from the recruitment of the donor. Therefore, diagnosis, collection of clinical data, follow-ups, and informed consent must take the long term into account. Interdisciplinary collaboration among neuropathologists, clinicians, ethicists, 
and IT specialists is indispensable, particularly at the recruitment stage, to making brain-bank research a successful endeavor. Secondly, as the obtaining, processing, and archiving of brain material is relatively labor-intensive, the sustainable funding and cost coverage for brain banks are crucial, and sensitive, issues. If public funding will not be sufficient to cover the long-term costs of BB, then future brain banks must find models for the adequate remuneration of their costs while simultaneously maintaining the notion that donation is altruistic. Thirdly, as with biobanks for rare diseases, there is a scarcity of well-characterized brain materials in general and tissues from certain regions in particular. Thus, the establishment of brain-bank networks with fair and effective allocation mechanisms is of the utmost importance. Finally, since the acquisition of whole brain material depends on the death of donors, there must be special requisites for their recruitment. In this regard, the role of the next of kin deserves further reflection. Stressing that research into neurodegenerative and psychiatric diseases is an important public good, some researchers not only question the family's right to overrule the donor's consent after his/her death but also call for a "a presumption in favor of donation" when the donor's wishes are unknown. ${ }^{10}$ This position, however, presumes that people trust research and the donation system. But, as this trust cannot be taken for granted, communication that strengthens the public understanding of brain donation may be more appropriate than legally discounting the family's concerns.

\section{Acknowledgments}

We would like to thank Walter Schulz-Schaeffer for all of his help with issues related to neuropathology and his critical reading of the manuscript. Moreover, we are indebted to Greg Sax for his editing. The collaboration for this project evolved from the Mentoring Program of the University Medical Center Göttingen, which is funded by the State of Lower Saxony (Germany), funding number 01 FP 09173 A. The project itself is funded by the Research Program of the University Medical Center, University of Goettingen.

\section{Disclosure}

The authors report no conflicts of interest in this work.

\section{References}

1. Kauffmann F, Cambon-Thomsen A. Tracing biological collections: between books and clinical trials. JAMA. 2008;299(19):2316-2318.

2. Hewitt R, Watson P. Defining biobank. Biopreserv Biobank. 2013;11(5): 309-315.

3. Lenk C, Hoppe N, Beier K, Wiesemann C. Human tissue research: A European perspective on the ethical and legal challenges. 1st ed. Oxford, New York: Oxford University Press; 2011.
4. Solbakk JH, Holm S, Hofmann B. The ethics of research biobanking. Dordrecht, London: Springer; 2009.

5. Artene S, Ciurea ME, Purcaru SO, et al. Biobanking in a constantly developing medical world. The ScientificWorldJournal. 2013;2013: 343275 .

6. Demiroglu SY, Skrowny D, Quade M, et al. Managing sensitive phenotypic data and biomaterial in large-scale collaborative psychiatric genetic research projects: practical considerations. Mol Psychiatry. 2012;17(12):1180-1185.

7. Buckow K, Quade M, Rienhoff O, Nussbeck SY. Changing requirements and resulting needs for IT-infrastructure for longitudinal research in the neurosciences. Neurosci Res. Epub August 22, 2014.

8. Nussbeck SY, Benson EE, Betsou F, Guadagni F, Lehmann S, Umbach N. Is there a protocol for using the SPREC? Biopreserv Biobank. 2013; 11(5):260-266.

9. Dedova I, Harding A, Sheedy D, et al. The Importance of Brain Banks for Molecular Neuropathological Research: The New South Wales Tissue Resource Centre Experience. Int J Mol Sci. 2009;10(1):366-384.

10. Harmon SH, McMahon A. Banking (on) the brain: From consent to authorisation and the transformative potential of solidarity. Med Law Rev. 2014;22(4):572-605.

11. Harper S. Economic and social implications of aging societies. Science. 2014;346(6209):587-591.

12. Di Luca M, Baker M, Corradetti R, et al. Consensus document on European brain research. The Eur J Neurosci. 2011;33(5):768-818.

13. de Oliveira KC, Nery FG, Ferreti RE, et al. Brazilian psychiatric brain bank: a new contribution tool to network studies. Cell Tissue Bank. 2012;13(2):315-326.

14. Stone K. Researchers take on a preventable dementia: brain bank is giving researchers new understanding of chronic traumatic encephalopathy. Ann Neurol. 2011;70(2):A11-A14.

15. Morgello S, Estanislao L, Simpson D, et al. HIV-associated distal sensory polyneuropathy in the era of highly active antiretroviral therapy: the Manhattan HIV Brain Bank. Arch Neurol. 2004;61(4):546-551.

16. Netherlands Brain Bank. Netherlands Brain Bank Progress Report 2011-2012; 2013. http://www.brainbank.nl/media/uploads/file/NBBPR1112.pdf. Accessed February 26, 2015.

17. Columbia Medicine [homepage on the Internet]. Neighbor J. New York Brain Bank: Deposits and Withdrawals That Make a Difference; 2014. Available from: http://www.columbiamedicinemagazine.org/features/ fall-2014/new-york-brain-bank. Accessed March 20, 2015.

18. Bell JE, Alafuzoff I, Al-Sarraj S, et al. Management of a twenty-first century brain bank: experience in the BrainNet Europe consortium. Acta Neuropathol. 2008;115(5):497-507.

19. Schmitt A, Bauer M, Heinsen H, et al. How a neuropsychiatric brain bank should be run: a consensus paper of Brainnet Europe II. J Neural Transm. 2007;114(5):527-537.

20. Kretzschmar H. Brain banking: opportunities, challenges and meaning for the future. Nat Rev Neurosci. 2009;10(1):70-78.

21. Ravid R. Standard Operating Procedures, ethical and legal regulations in BTB (Brain/Tissue/Bio) banking: what is still missing? Cell Tissue Bank. 2008;9(2):121-137.

22. Ravid R, Park YM. Brain banking in the twenty-first century: creative solutions and ongoing challenges. BSAM. 2014;2:17-27.

23. BrainNet Europe [homepage on the Internet]. Model Brain Bank Regulations; 2009. http://www.brainnet-europe.org/index. php?option $=$ com_content $\&$ view $=$ article $\&$ id $=105 \&$ Itemid $=105$. Accessed October 31, 2014.

24. McMahon A, Harmon SH. Banking (On) the Brain: A Report on the Legal and Regulatory Concerns. SCRIPT-ed. 2012;9(3):376-383.

25. BrainNet Europe [homepage on the Internet]. Code of Conduct; 2008. Available from: http://www.brainnet-europe.org/images/content/en/ media/code/code_of_conduct.pdf. Accessed March 22, 2015.

26. Klioueva NM, Rademaker MC, Dexter DT,et al. BrainNet Europe's Code of Conduct for brain banking. Available from: http://link.springer.com/ article/10.1007/s00702-014-1353-5/fulltext.html. Accessed March 22, 2015. 
27. Ravid R, Ikemoto K. Pitfalls and practicalities in collecting and banking human brain tissues for research on psychiatric and neulogical disorders. Fukushima J Med Sci. 2012;58(1):82-87.

28. Graeber MB. Twenty-first century brain banking: at the crossroads. Acta Neuropathol. 2008;115(5):493-496.

29. Hörnlimann B, Pauli G, Lemmer K, Beekes M, Mielke M. Prevention of the Transmission of Prion Diseases in Healthcare Settings. In: Hörnlimann B, Riesner D, Kretzschmar H, editors. Prions in Humans and Animals. Berlin: Walter de Gruyter; 2006:546-560.

30. Schwanke J, RienhoffO, Schulze TG, Nussbeck SY. Suitability of customer relationship management systems for the management of study participants in biomedical research. Methods Inf Med. 2013;52(4): 340-350.

31. Schmitt FA, Wetherby MM, Wekstein DR, Dearth CM, Markesbery WR. Brain donation in normal aging: procedures, motivations, and donor characteristics from the Biologically Resilient Adults in Neurological Studies (BRAiNS) Project. Gerontologist. 2001;41(6):716-722.

32. O'Grady G. Death of the teaching autopsy. BMJ. 2003;327(7418): 802-803.

33. Eatough V, Shaw K, Lees A. Banking on brains: insights of brain donor relatives and friends from an experiential perspective. Psychol Health 2012;27(11):1271-1290.

34. Olesen J, Baker MG, Freund T, et al. Consensus document on European brain research. J Neurol Neurosurg Psychiatry. 2006;77 Suppl 1 i1-i49.

35. Garrick T, Sundqvist N, Dobbins T, Azizi L, Harper C. Factors that influence decisions by families to donate brain tissue for medical research. Cell Tissue Bank. 2009;10(4):309-315.

36. Millar T, Walker R, Arango J, et al. Tissue and organ donation for research in forensic pathology: the MRC Sudden Death Brain and Tissue Bank. J Pathol. 2007;213(4):369-375.

37. Cruz-Sanchez FF, Mordini E, Ravid R. Ethical aspects to be considered in brain banking. Ann Ist Super Sanita. 1997;33(4):477-482.

38. Amarasinghe M, Tan H, Larkin S, et al. Banking the brain. Addressing the ethical challenges of a mental-health biobank. EMBO Rep. 2013 14(5):400-404.

39. Benes FM. Ethical issues in brain banking. Curr Opin Psychiatry. 2005; 18(3):277-283.

40. Boyes M, Ward P. Brain donation for schizophrenia research: gift, consent, and meaning. J Med Ethics. 2003;29(3):165-168.

41. Council of Europe [homepage on the Internet]. Convention for the Protection of Human Rights and Dignity of the Human Being with regard to the Application of Biology and Medicine: Convention on Human Rights and Biomedicine; 2007. Available from: http://conventions.coe int/Treaty/en/Treaties/html/164.htm. Accessed October 31, 2014.

42. World Medical Association [homepage on the Internet]. WMA Declaration of Helsinki - Ethical Principles for Medical Research Involving Human Subjects; 2013. Available from: http://www.wma net/en/30publications/10policies/b3/. Accessed October 31, 2014

43. Hulette CM. Brain banking in the United States. J Neuropathol Exp Neurol. 2003;62(7):715-722.

44. Council of Europe [homepage on the Internet]. Recommendation $\operatorname{Rec}(2006) 4$ of the Committee of Ministers to member states on research on biological materials of human origin; 2006. Available from: https:// wcd.coe.int/ViewDoc.jsp?id=977859. Accessed February 9, 2015.

45. Steinsbekk KS, Kåre Myskja B, Solberg B. Broad consent versus dynamic consent in biobank research: is passive participation an ethical problem? Eur J Hum Genet. 2013;21(9):897-902.

46. Faldum A, Pommerening K. An optimal code for patient identifiers. Comput Methods Programs Biomed. 2005;79(1):81-88.

47. Polman CH, Reingold SC, Banwell B, et al. Diagnostic criteria for multiple sclerosis: 2010 revisions to the McDonald criteria. Ann Neurol. 2011;69(2):292-302.

48. Wilcox AB, Gallagher KD, Boden-Albala B, Bakken SR. Research data collection methods: from paper to tablet computers. Med Care. 2012;50 Suppl:S68-S73.

49. Stone AA, Shiffman S, Schwartz JE, Broderick JE, Hufford MR. Patient non-compliance with paper diaries. BMJ. 2002;324(7347): 1193-1194.
50. Parkinson's UK Brain Bank. Donor Information Booklet. London; 2013. Available from: http://www.parkinsons.org.uk/sites/default/files/parkinsonsuk_brain_bank_information_booklet.pdf. Accessed February 25, 2015.

51. Azizi L, Garrick TM, Harper CG. Brain donation for research: strong support in Australia. J Clin Neurosci. 2006;13(4):449-452.

52. Wilkinson TM. Individual and family consent to organ and tissue donation: is the current position coherent? J Med Ethics. 2005;31(10): 587-590.

53. BrainNet Europe [homepage on the Internet]. Model information leaflet concerning registration for and brain donation to the brain bank; 2010. Available from: http://www.brainnet-europe.org/images/content/en/ media/d143_information_to_the_donor_20100620.doc. Accessed November 17, 2014

54. Zentrale Ethikkommission. Die (Weiter-)Verwendung von menschlichen Körpermaterialien für Zwecke medizinischer Forschung; 2003 [The (re-)use of human body materials for purposes of medical research]. Available from: http://www.zentrale-ethikkommission.de/downloads/ Koerpermat.pdf. Accessed October 31, 2014.

55. Human Tissue Authority UK. Code of practice 1: Consent; 2014. Available from: http://www.hta.gov.uk/_db/_documents/Code_of_ practice_1_-_Consent.pdf. Accessed November 17, 2014.

56. Vonsattel JP, Del Amaya MP, Cortes EP, Mancevska K, Keller CE. Twenty-first century brain banking: practical prerequisites and lessons from the past: the experience of New York Brain Bank, Taub Institute, Columbia University. Cell Tissue Bank. 2008;9(3):247-258.

57. Nussbeck SY, Skrowny D, O’Donoghue S, Schulze TG, Helbing K. How to design biospecimen identifiers and integrate relevant functionalities into your biospecimen management system. Biopreserv Biobank. 2014;12(3):199-205.

58. BrainNet Europe. Al-Sarraj S. Dementia Protocols; 2008. Available from: http://www.brainnet-europe.org/images/content/en/bilder/ Dementia_Protocols.pdf. Accessed November 17, 2014.

59. Schott JM, Reiniger L, Thom M, et al. Brain biopsy in dementia: clinical indications and diagnostic approach. Acta Neuropathol. 2010;120(3): 327-341.

60. Bell JE, Ironside JW. Principles and practice of 'high risk' brain banking. Neuropathol Appl Neurobiol. 1997;23(4):281-288.

61. Brown P, Wolff A, Gajdusek DC. A simple and effective method for inactivating virus infectivity in formalin-fixed tissue samples from patients with Creutzfeldt-Jakob disease. Neurology. 1990;40(6): 887-890.

62. Wemheuer WM, Wrede A, Gawinecka J, Zerr I, Schulz-Schaeffer WJ. Filtration of protein aggregates increases the accuracy for diagnosing prion diseases in brain biopsies. J Neuropathol Exp Neurol. 2013;72(8): 758-767.

63. Vonsattel JP, Del Amaya MP, Keller CE. Twenty-first century brain banking. Processing brains for research: the Columbia University methods. Acta Neuropathol. 2008;115(5):509-532.

64. Alafuzoff I, Thal DR, Arzberger T, et al. Assessment of beta-amyloid deposits in human brain: a study of the BrainNet Europe Consortium. Acta Neuropathol. 2009;117(3):309-320.

65. Huey ED. Clinical-pathological agreement in dementing disorders: embracing the complexity. Tremor Other Hyperkinet Mov (NY). 2014;4:268.

66. Adler $\mathrm{CH}$, Beach TG, Hentz JG, et al. Low clinical diagnostic accuracy of early vs advanced Parkinson disease: clinicopathologic study. Neurology. 2014;83(5):406-412.

67. Beach TG, Monsell SE, Phillips LE, Kukull W. Accuracy of the clinical diagnosis of Alzheimer disease at National Institute on Aging Alzheimer Disease Centers, 2005-2010. J Neuropathol Exp Neurol. 2012;71(4): 266-273.

68. Morgello S, Gelman BB, Kozlowski PB, et al. The National NeuroAIDS Tissue Consortium: a new paradigm in brain banking with an emphasis on infectious disease. Neuropathol Appl Neurobiol. 2001;27(4): 326-335.

69. Nature.com blogs [homepage on the Internet]. Maxmen A. Brain thaw at Harvard repository. Available from: http://blogs.nature.com/ news/2012/06/brains-thaw-at-harvard-repository.html. Accessed October 29, 2014. 
70. Reuters [homepage on the Internet]. Clarke T. Hospital freezer fault destroys crucial brain data. Available from: http://www.reuters. com/article/2012/06/11/us-brain-research-damage-idUSBRE85A1EG20120611. Accessed February 25, 2015.

71. Samarasekera N, Salman RA, Huitinga I, et al. Brain banking for neurological disorders. The Lancet Neurol. 2013;12(11):1096-1105.

72. Lenk $\mathrm{C}$, Beier $\mathrm{K}$. Is the commercialisation of human tissue and body material forbidden in the countries of the European Union? $\mathrm{J} \mathrm{Med}$ Ethics. 2012;38(6):342-346.

73. Wei B, Simpson RM. Digital pathology and image analysis augment biospecimen annotation and biobank quality assurance harmonization. Clin Biochem. 2014;47(4-5):274-279.
74. Abdelmoula WM, Carreira RJ, Shyti R, et al. Automatic registration of mass spectrometry imaging data sets to the Allen brain atlas. Anal Chem. 2014;86(8):3947-3954.

75. Zaldivar A, Krichmar JL. Allen Brain Atlas-Driven Visualizations: a web-based gene expression energy visualization tool. Front Neuroinform. 2014;8:51.

76. Majka P, Kowalski JM, Chlodzinska N, Wójcik DK. 3D brain atlas reconstructor service - online repository of three-dimensional models of brain structures. Neuroinformatics. 2013;11(4):507-518.
Journal of Biorepository Science for Applied Medicine

\section{Publish your work in this journal}

Journal of Biorepository Science for Applied Medicine is an international, peer-reviewed, open access journal that focuses on new developments and advances in the emergin and evolving field of biorepository science. This includes biospecimen procurement, processing, preservation, and banking for application to applied medicine. The Journal invites submission of manuscripts which address these aspects in addition to systems logic, clinical throughput and ethical issues pertaining to application of biorepositories

\section{Dovepress}

and their affects on clinical medicine. The journal is characterized by the rapid reporting of reviews, original research, methodologies, technologies and analytics in this subject area. The manuscript management system is completely online and includes a very quick and fair peer-review system, which is all easy to use. Visit http://www.dovepress. com/testimonials.php to read real quotes from published authors.

Submit your manuscript here: http://www.dovepress.com/journal-of-biorepository-science-for-applied-medicine-journal 